\title{
Optimal Acknowledgement Strategies for Multichannel Reservation Multi-access Schemes under Rayleigh Fading
}

\author{
Atul Maharshi, Lang Tong* \\ School of Electrical Engineering \\ Cornell University \\ Ithaca, NY 14853 \\ \{atul,ltong\}@ece.cornell.edu
}

\author{
Anathram Swami \\ Army Research Lab \\ 2800 Powder Mill Road \\ Adelphi, MD 20783 \\ aswami@arl.army.mil
}

\begin{abstract}
A reservation-based medium access control (MAC) scheme is considered where users reserve data channels through a slotted-ALOHA procedure. The base station grants access to users in a Rayleigh fading environment using detectors that utilize measurements at the physical layer and system information at the MAC layer. The performance analysis is based on a Markov Chain formulation and optimal performance obtainable in the given scenario is evaluated. An acknowledgement strategy based on costoptimized Bayesian detectors which achieves this optimal performance is presented along with strategies based on other detectors such as MAP, ML and UMP. The delay/channel-utilization trade-off is considered for various system parameters.
\end{abstract}

\section{INTRODUCTION}

Detection plays a key role in Medium Access Control (MAC) of random access networks. In UMTS-WCDMA [6], for example, a random access scheme is used for channel (code) reservation. Users request transmissions by sending a signature randomly chosen from a pool of available codes. The base station grants or denies their transmissions based on the measured signal strength. A collision occurs if multiple $(>1)$ users send requests for a code and that code is acknowledged by the base station by mistake. On the other hand, if a code is acknowledged without an user request, the code is mistakenly taken out of the pool of available codes for other users, which causes inefficient channel (code) utilization, heavier traffic, and more frequent collisions in other channels.

It is not obvious that the classical approach to optimal detection naturally leads to optimal MAC performance. Here, we must take into account properties of the arrival process and the impact of collision on throughput and delay. On this, the literature is scarce; only a few ad hoc schemes have been reported $[2,3,7]$.

Motivated by the idea of cross layer design of signal detection and MAC, we considered detection and acknowledgement strategies for a reservation random access network with transmissions undergoing flat Rayleigh fading in [8]. We exploited information from measurements at the physical layer, the traffic statistics, and the network states at the MAC layer in the design of detectors. Here, we relax the assumption of geometrically distributed packet lengths, and consider a scenario where users transmit fixed-length packets.

*This work was supported in part by the Multidisciplinary University Research Initiative (MURI) under the office of Naval Research Contract N00014-00-1-0564 and the ARL CTA on Communications and Networks.
The paper is organized as follows. We describe the system model in Sec.II where basic functions and assumptions are presented separately for mobiles and base stations. In Sec.III, a throughput analysis is presented using a Markov chain formulation. Detector design is considered in Sec.IV where we present several detectors optimized for throughput and also discuss the issue of improving the throughput by means of multiple measurements. Finally, simulation results are discussed in Sec.V.

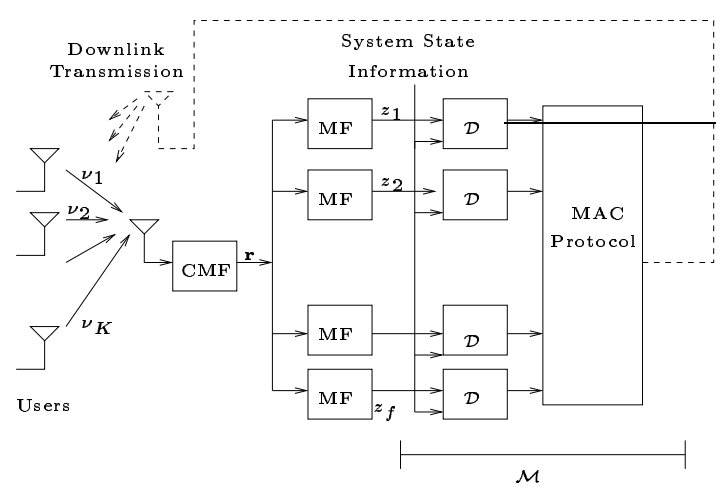

Fig. 1: A reservation-based random access in CDMA. CMF: Chipmatched-filtering and sampling. MF: Code matched filter.

\section{System Model}

We use base station to include the usual cellular base station, as well as clusterheads or privileged nodes in an ad hoc network, that have multiple codes.

\section{A The Mobile Stations}

The random access scheme is based on the slotted ALOHA channel reservation. At the beginning of slot $t$, the base station broadcasts a set $\mathcal{C}_{t}$ of available orthogonal preamble signatures for uplink reservation. An interested user transmits a randomly selected signature from $\mathcal{C}_{t}$ and waits for an acknowledgement. If a positive acknowledgement is received, the user proceeds to transmit data using an orthogonal code having a one-to-one relationship with the preamble signature; the data transmission lasts for a fixed duration of $L$ slots. If a channel is acknowledged when two or more users are attempting access, a collision occurs and the channel becomes locked, i.e., it is unavailable to the other users even though the channel is not contributing to the throughput. We further note that a channel might get locked when the base station transmits an ACK even when no user is attempting access. Regardless of the way a channel gets occupied, we assume that the channel remains unavailable to the other users for a length of $L$ slots. 
In case no acknowledgement is received, the user backs off and retries after a random delay. We assume that no preamble power ramping is carried out i.e., a user does not increase power on retries.

We assume that the access attempts that include new arrivals as well as retries lead to a Poisson process with intensity $\lambda$ attempts/slot. See [2, Ch. 3-4],[4] for comments regarding the Poisson arrival process.

\section{$B$ The Base Station}

After announcing the available preamble signatures $\mathcal{C}_{t}$, the base station performs matched filtering for each code in $\mathcal{C}_{t}$. The output of each matched filter (see Fig. 1) is used in some form of hypothesis testing (denoted as $\mathcal{D}$ ), and the MAC protocol makes decisions on acknowledgement based on the outcome of the test.

We emphasize that the size of $\mathcal{C}_{t}$ varies from slot to slot, which makes the attempt rate time varying at each channel (even though the overall attempt rate is constant). The fluctuation of the available signatures makes detection thresholds time varying. This makes the optimal detection problem nontrivial.

\section{MAC PERFORMANCE}

In this section, we show that the proposed MAC induces a Markov Chain (MC) structure which facilitates throughput analysis. The MC will be shown to be statioanry, and throughput will be seen to depend upon $\alpha_{f}\left(=1-\beta_{f}\right)$ and $\gamma_{f} ; \alpha_{f}$ is the conditional probability of acknowledging a channel, given that there are $f$ free channels; $\gamma_{f}$ is the conditional probability that a channel is ACKed and exactly one user is attempting access on that channel. Parameters $\alpha_{f}, \gamma_{f}$ depend upon the detector used at the PHY layer to detect the presence/absence of exactly one user requesting a given channel. In the proposed joint $\mathrm{PHY} / \mathrm{MAC}$ design ( $\mathcal{M}$ in fig.1), we optimize detector parameters to maximize throughput.

A channel once occupied remains so for a duration of $L$ slots. The system, thus, has a memory of $L$ slots. We define the state vector as

$$
\mathbf{n}_{t}=\left[n_{t-L+1}, \cdots, n_{t}\right] \in \mathcal{S}
$$

where $n_{t}$ is the number of newly locked channels at the beginning of slot $t, \mathcal{S}$ being the state space. Definition of the states in this manner can indeed be seen to result in a $\mathrm{MC}$, with the transition probabilities being governed by the detector characteristics and the traffic statistics. The MC is finite as $n_{t} \leq N_{c}$ for all $t$.

The number of free channels, i.e., the channels that are being contended for, in slot $t$ is $f=N_{c}-\sum_{i=0}^{L-1} n_{t-i}$. The traffic statistics as seen by a detector for a given free code changes with the number of free codes. Conditioned on the number of free codes and the detector characteristics, and given the traffic statistics we can compute the probabilities $\alpha_{f}$ and $\gamma_{f}$. We shall assume that given the channel state, the detectors act independently of each other.

\section{A The case of $N_{c}=2$}

To provide a flavor of the investigation being pursued and the difficulties thrown up in evaluation, we turn our attention to the principal focus of this paper, the case when $N_{c}=2$. This case yields readily to analysis and it is possible to derive expressions for throughput for any given $L$. For $N_{c}=2$, we can partition the state space into four groups: $\mathcal{S}=\mathcal{S}_{0} \oplus \mathcal{S}_{1} \oplus$ $\mathcal{S}_{2} \oplus \mathcal{S}_{3} . \mathcal{S}_{0}$ contains the state $S_{0} . \mathcal{S}_{0} \triangleq\{[0 \ldots 0]\} . \mathcal{S}_{1}$ contains sates with a single locked channel:

$$
\mathcal{S}_{1} \triangleq\{[0 \ldots 01], \ldots,[10 \ldots 0]\}=\left\{S_{1}, \ldots, S_{L}\right\} .
$$

$\mathcal{S}_{2}$ contains states with two simultaneously locked channels:

$$
\mathcal{S}_{2} \triangleq\{[0 \ldots 02], \ldots,[20 \ldots 0]\}=\left\{S_{L+1}, \ldots, S_{2 L}\right\} .
$$

$\mathcal{S}_{3}$ contains states with two channels locked at different slots

$$
\begin{aligned}
\mathcal{S}_{3} & \triangleq\{[0 \ldots, 011],[0, \ldots 0101], \ldots,[110 \ldots 0]\} \\
& =\left\{S_{2 L+1}, \ldots, S_{3 L-1}, S_{3 L}, \ldots, S_{|\mathcal{S}|-1}\right\}
\end{aligned}
$$

Note that $|\mathcal{S}|=(L+1)(L+2) / 2$. The partioning above has been done such that there is a 'translational invariance' between the components describing the states within each group. For example, with $L=3$, [011] and [101] belong to the same group $-\mathcal{S}_{3}$. The stationary distribution denoted by $\pi=\left[\pi_{0}, \cdots, \pi_{|\mathcal{S}|-1}\right]$ is given by:

$$
\pi_{i}= \begin{cases}\frac{1}{L \alpha_{2}^{2}+2 L \alpha_{2} \beta_{2} / \beta_{1}+2\left(\begin{array}{c}
L \\
2
\end{array}\right) \alpha_{1} \alpha_{2} \beta_{2} / \beta_{1}+1} \triangleq p_{0} & i=0 \\
2 \alpha_{2} \beta_{2} \pi_{0} / \beta_{1} \triangleq p_{1} & 1 \leq i \leq L \\
\alpha_{2}^{2} \pi_{0} \triangleq p_{2} & L<i \leq 2 L \\
2 \alpha_{1} \alpha_{2} \beta_{2} / \beta_{1} \pi_{0} \triangleq p_{3} & 2 L<i<|\mathcal{S}|\end{cases}
$$

\section{$B$ Throughput and Channel Utilization}

Having obtained the stationary distribution one can now obtain figures of merit for network performance. We consider two figures of merit, throughput and channel utilization. Throughput is defined as the average number of successful access attempts per slot and channel utilization as the number of successful transmissions per slot per channel. Channel utilization can also be thought of as the fraction of the slots that actually get utilized for data transmission. The throughput can be written as:

$$
\eta=2 \gamma_{2} \pi_{0}+\sum_{i=1}^{L} \gamma_{1} \pi_{i}=2 \gamma_{2} p_{0}+L \gamma_{1} p_{1}
$$

where it can be seen that $2 \gamma_{2}$ is the expected number of access attempts that are successful when in state $S_{0}$ and $\gamma_{1}$ is the corresponding value when in a state belonging to $\mathcal{S}_{1}$. Each successful user occupies the channel for $L$ slots. Thus, the average number of successful transmissions per slot per channel is given by:

$$
\zeta=L \eta / N_{c} .
$$

Thus, for a given $L$, the detector strategy that maximizes $\eta$ also maximizes the channel occupancy.

For given $L$ and $\lambda$, we have the following optimization problem: find $\mathcal{M}_{0}$ such that

$$
\mathcal{M}_{0}(\lambda)=\arg \max _{\mathcal{M}} \eta_{\lambda}(\mathcal{M})
$$

$\mathcal{M}$ is a combination of the physical layer detector and the ACK strategy. In the next section we consider some strategies based on various detectors available in the classic literature. Our aim is to obtain the probabilities $\alpha_{f}$ and $\gamma_{f}$, given the type of detector and its operating point, so that we could later compare performance under fading channel conditions. 


\section{$C$ Behaviour for large $L$}

The channel utilization can be expected to go up as $L$ increases. However, the utilization obtained need not be arbitrarily close to the ideal. The limit as $L$ grows large depends on the fading conditions and the detector ROCs. Substituting the expression for $\eta$ we can directly evaluate the limit for (8) as $L \rightarrow \infty$. Note that we get two different limits for the cases $\alpha_{1} \neq 0$ and $\alpha_{1}=0$. Optimizing over the ROCs we get:

$$
\zeta \rightarrow \max \left(\max \left(\frac{\gamma_{1}}{\alpha_{1}}\right), \max \left(\frac{\gamma_{2}}{\alpha_{2}\left(1+\beta_{2}\right)}\right)\right) .
$$

\section{The Detector}

The detection considered here differs from the classical detection problem in two aspects. First, our objective is to maximize the network throughput. Second, the detector needs to exploit the system state information (e.g., number of available channels). The challenge arises from the fact that detection errors affect the system state which in turn affects the statistics of the incoming traffic. Detection errors therefore should not be treated in the classical way.

\section{A The Signal Model}

Consider the model depicted in Fig.1. Each available channel has a detector associated with it. The detector takes as its input the sampled chip-matched filtered signal. We assume that the user signals undergo iid Rayleigh flat fading. Assume that $f$ channels are available and $K$ users contend for reservation. The sampled output of the chip-matched filtered can be written as:

$$
\mathbf{r}=\sum_{k=1}^{K} \nu_{k} \mathbf{s}_{k}+\mathbf{w}
$$

where $\nu_{k}$ are the complex amplitudes which are i.i.d. with distribution $\mathcal{C} N\left(0, \sigma_{d}^{2} / 2 N^{2}\right), N$ is the signature length in chips, $\sigma_{d}^{2}$ the SNR. All the symbols in bold font denote vectors of length $N$. The signatures, $\mathbf{s}_{k}$, belong to the set of available orthogonal signatures, $\mathcal{C}_{t}=\left\{\mathbf{c}_{1}, \mathbf{c}_{2}, \ldots, \mathbf{c}_{f}\right\}$. The elements of $\mathcal{C}_{t}$ have a one-to-one relationship with the set of available channels and $\mathbf{c}_{i}^{H} \mathbf{c}_{i}=N$ for $i=1,2, \ldots, f$ ( $H$ denotes the Hermitian operator). The noise $\mathbf{w}$ is AWGN with distribution $\mathcal{C} N(0,1 / 2 N \mathbf{I})$.

At the $i^{t h}$ detector, decorrelating with the signature $\mathbf{c}_{i}$ we get:

$$
\begin{aligned}
z_{i} & =\mathbf{c}_{i}^{H} \mathbf{r} \\
& =\sum_{k: \mathbf{s}_{k}=\mathbf{c}_{i}} N \alpha_{k}+w_{i}=\sqrt{\theta_{i}} x_{i}+w_{i}
\end{aligned}
$$

where $\sqrt{\theta_{i}} x_{i} \sim \mathcal{C} N\left(0, \theta_{i} \sigma_{d}^{2} / 2\right)$ is the component due to $\theta_{i}$ users selecting signature $\mathbf{c}_{i}$ and $w_{i} \sim \mathcal{C} N(0,1 / 2)$. At this point we may drop the subscript $i$ for the detector, because given $f$, the working of each detector is identical to the rest. Notice that $|z|^{2}$ is a sufficient statistic for $\theta$, and that $|z|^{2}$ has an exponential distribution. For ease of notation, we will let $y=$ $|z|^{2}$ in the rest of the paper. Based on the above statistic the detector must make a decision on whether or not a single user is attempting access, i.e., is $\theta=1$. The problem is to make this decision so as to improve the performance at the network level.

Formally, consider the following [1] parameter set defined by the user indices $\Lambda=\{0,1,2,3, .$.$\} ; random parameter, \Theta$, taking on values in $\Lambda$. The realization, $\theta$, is the number of users attempting reservation of the same channel. The decision rule should be based on $y$. We know that the conditional distribution of $y$ given $\Theta=\theta$ is

$$
p(y \mid \theta)=\frac{1}{\theta \sigma_{d}^{2}+1} \exp \left(\frac{-y}{\theta \sigma_{d}^{2}+1}\right) .
$$

Also, since the arrivals are Poisson, given that $f$ channels are free, the access attempt rate for a particular channel is $\lambda_{f}=\lambda / f$. Thus, the prior probability for $\theta$ given the arrival rate can be written as

$$
w(\theta)=\exp \left(-\lambda_{f}\right) \frac{\left(\lambda_{f}\right)^{\theta}}{\theta !} .
$$

\section{$B$ The Bayesian Detector}

We first consider the MAC protocol based on the binary hypothesis on the number of users transmitting a particular preamble. Specifically,

$$
\begin{array}{ll}
H_{0} & : \quad \theta \in\{0,2,3, \cdots\} \triangleq \Lambda_{0} \\
H_{1} & : \quad \theta \in\{1\} \triangleq \Lambda_{1} .
\end{array}
$$

Notice that $H_{0}$ is a composite hypothesis.

The Bayesian detector has the form

$$
\delta(y)=\left\{\begin{array}{lll}
1 & & > \\
0 \text { or } 1 & \frac{P\left(\Theta \in \Lambda_{1} \mid y\right)}{P\left(\Theta \in \Lambda_{0} \mid y\right)} & = \\
0 & & <
\end{array} \rho_{f}\right.
$$

where $\rho_{f}$ is the 'cost-ratio' dependent on the number of free channels as noted above. Now, since the cost-ratios are actually unknown, we might try a search for the optimal $\rho_{f}$ with $f=1,2, \ldots, N_{c}$. For the present model, we have [8]:

$$
\frac{P\left(\Theta \in \Lambda_{1} \mid y\right)}{P\left(\Theta \in \Lambda_{0} \mid y\right)}=\frac{\frac{1}{\sigma_{d}^{2}+1} \exp \left(\frac{-y}{\sigma_{d}^{2}+1}\right) \lambda_{f}}{\sum_{\theta \neq 1} \frac{1}{\theta \sigma_{d}^{2}+1} \exp \left(\frac{-y}{\theta \sigma_{d}^{2}+1}\right) \frac{\lambda_{f}^{\theta}}{\theta !}} .
$$

Given cost-ratio $\rho_{f}$ and access rate $\lambda_{f}$, we can numerically determine the decision regions, $\Gamma_{1}\left(\rho_{f}, \lambda_{f}\right)$ and $\Gamma_{0}\left(\rho_{f}, \lambda_{f}\right)$ corresponding to the two hypotheses. The decision regions are of the form:

$$
\begin{array}{cc}
y \in \Gamma_{1}\left(\rho_{f}, \lambda_{f}\right) & \text { if } \tau_{1}\left(\rho_{f}, \lambda_{f}\right) \leq y \leq \tau_{2}\left(\rho_{f}, \lambda_{f}\right) \\
y \in \Gamma_{0}\left(\rho_{f}, \lambda_{f}\right) & \text { otherwise }
\end{array}
$$

where $\tau_{1}\left(\rho_{f}, \lambda_{f}\right)$ and $\tau_{2}\left(\rho_{f}, \lambda_{f}\right)$ can be interpreted as power thresholds based on which the detector makes its decisions. Intuitively, we would expect the decision regions to be of the form given in (19), so that power falling below the lower threshold corresponds to the case of no user attempting access, while power falling above the upper threshold corresponds to the case of two or more users attempting access.

Having obtained the decision regions, we can now obtain the event probabilities needed for the MC. Let $\tau_{1 f}=$ $\tau_{1}\left(\lambda_{f}, \rho_{f}\right)$ and $\tau_{2 f}=\tau_{2}\left(\lambda_{f}, \rho_{f}\right)$ be the lower and upper threhsolds given that $f$ channels are free. From the thresholds we can compute the probabilities needed in the MC:

$$
\begin{aligned}
& \alpha_{f}\left(\lambda_{f}, \rho_{f}\right)=\sum_{n=0}^{\infty} \frac{e^{-\lambda_{f}} \lambda_{f}^{n}}{n !}\left(e^{-\tau_{1 f} /\left(\theta \sigma_{d}^{2}+1\right)}-e^{-\tau_{2 f} /\left(\theta \sigma_{d}^{2}+1\right)}\right) \\
& \gamma_{f}\left(\lambda_{f}, \rho_{f}\right)=e^{-\lambda_{f}} \lambda_{f}\left(e^{-\tau_{1 f} /\left(\sigma_{d}^{2}+1\right)}-e^{-\tau_{2 f} /\left(\sigma_{d}^{2}+1\right)}\right) .
\end{aligned}
$$


Optimization now involves searching for the optimal vector $\left(\bar{\rho}=\left(\rho_{1}, \rho_{2}\right)\right)$ of cost-ratios:

$$
\bar{\rho}_{0}(\lambda)=\arg \max _{\bar{\rho}} \eta_{\lambda}(\bar{\rho})
$$

\section{The Multi-hypotheses MAP}

In [8], we considered the Multihypotheses MAP detector for which, unlike the Bayesian detector, the thresholds can be expressed in closed form. $H_{1}$ is held to be true when $\theta=1$ has the maximum a posteriori probability amongst all $\theta \in \Theta$ i.e., when:

$$
\arg \max _{\theta} p_{\theta}(y) w(\theta)=1 .
$$

For the Multi-hypotheses MAP detector the two thresholds depending on the arrival rate, $\tau_{1}\left(\lambda_{f}\right)$ and $\tau_{2}\left(\lambda_{f}\right)$ are determined by:

$$
\tau_{1}\left(\lambda_{f}\right)=\max \left\{\tilde{\tau}_{1}, 0\right\}, \tau_{2}\left(\lambda_{f}\right)=\inf \left\{\tilde{\tau}_{\theta}: \theta>1\right\}
$$

where

$$
\begin{aligned}
& \tilde{\tau}_{1}\left(\lambda_{f}\right)=\frac{\sigma_{d}^{2}+1}{\sigma_{d}^{2}} \log \left(\frac{\sigma_{d}^{2}+1}{\lambda_{f}}\right) \\
& \tilde{\tau}_{\theta}\left(\lambda_{f}\right)=\frac{\left(\sigma_{d}^{2}+1\right)\left(\theta \sigma_{d}^{2}+1\right)}{(\theta-1) \sigma_{d}^{2}} \log \left(\frac{\left(\theta \sigma_{d}^{2}+1\right) \theta !}{\lambda_{f}^{(\theta-1)}\left(\sigma_{d}^{2}+1\right)}\right)
\end{aligned}
$$

The probabilities $\alpha_{f}$ and $\gamma_{f}$ can now be computed to obtain the throuhgput. Since the thresholds are fixed directly by $\sigma_{d}^{2}$ and $\lambda_{f}$, this detector does not involve any optimization. One problem is that if $\lambda_{f}$ is large enough, $\tau_{2}$ could become negative.

\section{A Single Threshold Detector}

Single threshold detectors acknowledge a channel when the power exceeds a given threshold (the upper threshold $\tau_{2}=\infty$, which is essentially equivalent to assuming that the SNR is high). The detectors discussed in the literature available on $\mathrm{RACH}$ [3] belong to this class. In this case, we want to find the optimal among the $\bar{\tau}=\left(\tau_{s 1}, \tau_{s 2}\right)$, so that

$$
\bar{\tau}_{0}(\lambda)=\arg \max _{\bar{\tau}} \eta_{\lambda}(\bar{\tau}) .
$$

Given $\bar{\tau}$, we can obtain $\alpha_{f}$ and $\gamma_{f}$ required in the throughput expression.

\section{E UMP and $M L$ detectors}

In determining the thresholds for the Bayesian and Multihypotheses MAP, we require knowledge of the traffic statistics. We could use detectors which do not require prior probabilities when the knowledge of traffic statistics becomes unreliable. A Uniformly Most Powerful (UMP) test with parameter $\mu$ given in [5], does not require the priors to be known. The test can be written as:

$$
\delta(y)=\left\{\begin{array}{lc}
1 & \tau_{1}(\mu)<y<\tau_{2}(\mu) \\
0 \text { or } 1 & y=\tau_{1}(\mu) \text { or } y=\tau_{2}(\mu) \\
0 & y<\tau_{1}(\mu) \text { or } y>\tau_{2}(\mu)
\end{array}\right.
$$

where $\tau_{1}$ and $\tau_{2}$ satisfy:

$$
E(\delta(y) \mid \theta=0)=E(\delta(y) \mid \theta=2)=\mu .
$$

The condition above leads to the following expressions from which we can evaluate the thresholds:

$$
\begin{aligned}
\left(\mu+e^{-\tau_{2}}\right)^{1 /\left(2 \sigma^{2}+1\right)} & =\mu+e^{-\tau_{2} /\left(2 \sigma^{2}+1\right)} \\
e^{-\tau_{1}} & =\mu+e^{-\tau_{2}} .
\end{aligned}
$$

We can then compute the throughput having obtained $\alpha_{f}$ and $\gamma_{f}$. Again, the parameter, $\mu$, might depend on the number of free channels. The search must, therefore, be made over $\bar{\mu}=\left(\mu_{1}, \mu_{2}\right)$. The optimization involves finding:

$$
\bar{\mu}_{o}(\lambda)=\arg \max _{\bar{\mu}} \eta_{\lambda}(\bar{\mu}) .
$$

We could also use Maximum Likelihood test for multihypotheses to determine thresholds when the priors are not known. The thresholds for the ML detector are given by:

$$
\begin{aligned}
\tau_{1}\left(\lambda_{f}\right) & =\frac{\sigma_{d}^{2}+1}{\sigma_{d}^{2}} \log \left(\sigma_{d}^{2}+1\right) \\
\tau_{2}\left(\lambda_{f}\right) & =\frac{\left(\sigma_{d}^{2}+1\right)\left(2 \sigma_{d}^{2}+1\right)}{\sigma_{d}^{2}} \log \left(\frac{2 \sigma_{d}^{2}+1}{\sigma_{d}^{2}+1}\right) .
\end{aligned}
$$

As in the case of the Multihypotheses MAP, we do not have any degrees of freedom to optimize throughput. The number of free channels immediately fixes $\alpha_{f}$ and $\gamma_{f}$.

\section{F Multiple Measurements}

We expect throughput to increase with SNR. However, under Rayleigh fading, the throughput saturates, without reaching the ideal value. For a high SNR, we can only expect to make no error in judging the presence or absence of user(s). However, errors will still be made in distinguishing the presence or absence of exactly one user.

Multiple independent measurements can improve the performance of our detectors. Such multiple measurements could be obtained in the same slot or be spread out over consecutive slots depending on how fast the fading occurs.

Let the sampled, despread and match-filtered received vector obtained after $n$ measurements be $\mathbf{z}=\left[\begin{array}{lll}z_{1} & z_{2} \ldots z_{n}\end{array}\right], z_{i}$ 's are i.i.d. $\mathcal{C N}\left(0, \theta \sigma_{d}^{2}+1\right)$ where $\sigma_{d}^{2}$ is the SNR (the number of users attempting access is assumed to be $\theta$ ). Conditioned on $\theta,\left|z_{i}\right|^{2} \sim \exp \left(1 /\left(\theta \sigma_{d}^{2}+1\right)\right)$, the conditional distribution of $y$ is given by:

$$
p(y \mid \Theta=\theta)=\frac{y^{(n-1)}}{\left(\theta \sigma_{d}^{2}+1\right)^{n}(n-1) !} e^{\left(\frac{-y}{\theta \sigma_{d}^{2}+1}\right)} .
$$

And thus, the sufficient statistic is the sum of squared power of the received components -

$$
y=T(\mathbf{z})=\sum_{i=1}^{n}\left|z_{i}\right|^{2} .
$$

The ratio of aposteriori probabilities for the Bayesian detector is given by:

$$
\frac{P\left(\Theta \in \Lambda_{1} \mid y\right)}{P\left(\Theta \in \Lambda_{0} \mid y\right)}=\frac{\frac{1}{\left(\sigma_{d}^{2}+1\right)^{n}} \exp \left(\frac{-y}{\sigma_{d}^{2}+1}\right) \lambda_{f}}{\sum_{\theta \neq 1} \frac{1}{\left(\theta \sigma_{d}^{2}+1\right)^{n}} \exp \left(\frac{-y}{\theta \sigma_{d}^{2}+1}\right) \frac{\lambda_{f}^{\theta}}{\theta !}}
$$

The thresholds for the Multihypotheses MAP detector are given by:

$$
\begin{aligned}
& \tilde{\tau}_{1}\left(\lambda_{f}\right)=\frac{\sigma_{d}^{2}+1}{\sigma_{d}^{2}} \log \left(\frac{\left(\sigma_{d}^{2}+1\right)^{n}}{\lambda_{f}}\right) \\
& \tilde{\tau}_{\theta}\left(\lambda_{f}\right)=\frac{\left(\sigma_{d}^{2}+1\right)\left(\theta \sigma_{d}^{2}+1\right)}{(\theta-1) \sigma_{d}^{2}} \log \left(\frac{\left(\theta \sigma_{d}^{2}+1\right)^{n} \theta !}{\lambda_{f}^{(\theta-1)}\left(\sigma_{d}^{2}+1\right)^{n}}\right) .
\end{aligned}
$$




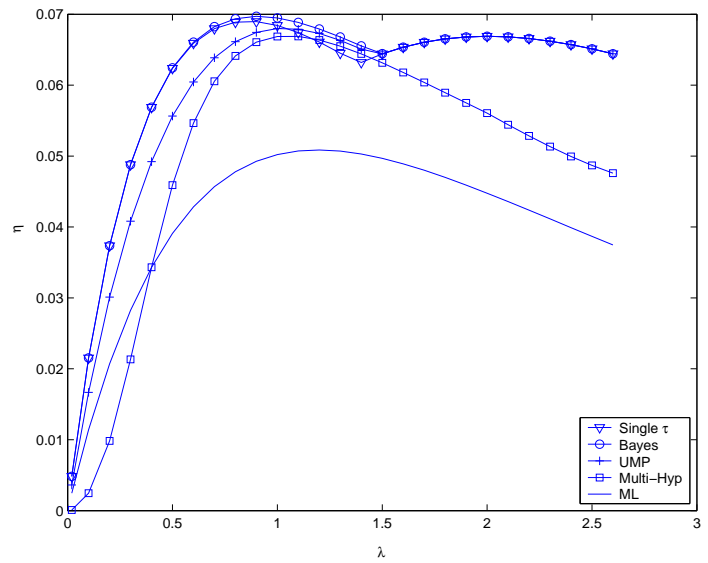

Fig. 2: Normalized Throughput v/s Arrival rate for various detectors with $\mathrm{L}$ equal to $10, \mathrm{SNR}=0 \mathrm{~dB}(\mathrm{left})$.

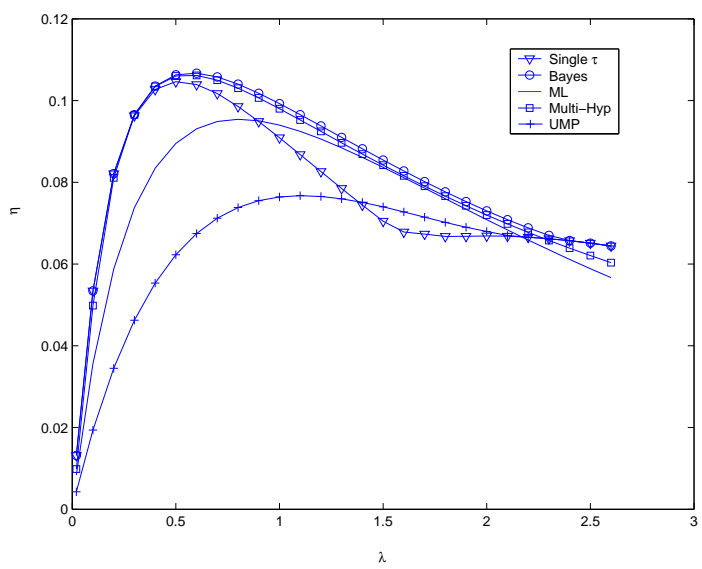

Fig. 3: Normalized Throughput v/s Arrival rate for various detectors with $\mathrm{L}$ equal to $10, \mathrm{SNR}=10 \mathrm{~dB}$.

and

$$
\tau_{1}=\max \left\{\tilde{\tau}_{1}, 0\right\}, \quad \tau_{2}=\inf \left\{\tilde{\tau}_{\theta}: \theta>1\right\}
$$

Having obtained the two thresholds, the probabilities required for evaluating $\eta$ can be obtained from:

$$
\begin{aligned}
\gamma_{f}= & \lambda_{f} e^{-\lambda_{f}}\left(e^{-\tau_{1 f} /\left(\sigma_{d}^{2}+1\right)}\left(\sum_{j=0}^{n-1} \frac{\left(\tau_{1 f} /\left(\sigma_{d}^{2}+1\right)\right)^{j}}{j !}\right)\right. \\
& \left.-e^{-\tau_{2 f} /\left(\sigma_{d}^{2}+1\right)}\left(\sum_{j=0}^{n-1} \frac{\left(\tau_{2 f} /\left(\sigma_{d}^{2}+1\right)\right)^{j}}{j !}\right)\right) \\
\alpha_{f}= & \sum_{\theta=0}^{\infty} e^{-\lambda_{f}}\left(e^{-\tau_{1 f} /\left(\theta \sigma_{d}^{2}+1\right)}\left(\sum_{j=0}^{n-1} \frac{\left(\tau_{1 f} /\left(\theta \sigma_{d}^{2}+1\right)\right)^{j}}{j !}\right)\right. \\
& \left.-e^{-\tau_{2 f} /\left(\theta \sigma_{d}^{2}+1\right)}\left(\sum_{j=0}^{n-1} \frac{\left(\tau_{2 f} /\left(\theta \sigma_{d}^{2}+1\right)\right)^{j}}{j !}\right)\right) \frac{\left(\lambda_{f}\right)^{\theta}}{\theta !} .
\end{aligned}
$$

\section{Numerical Results}

In this section we present the results of numerical evaluation of the throughput and channel utilization obtained with the various detectors. We will also consider aspects such as the dependence of throughput on the SNR, the packet length, $L$, and multiple measurements. Finally, we will look into the

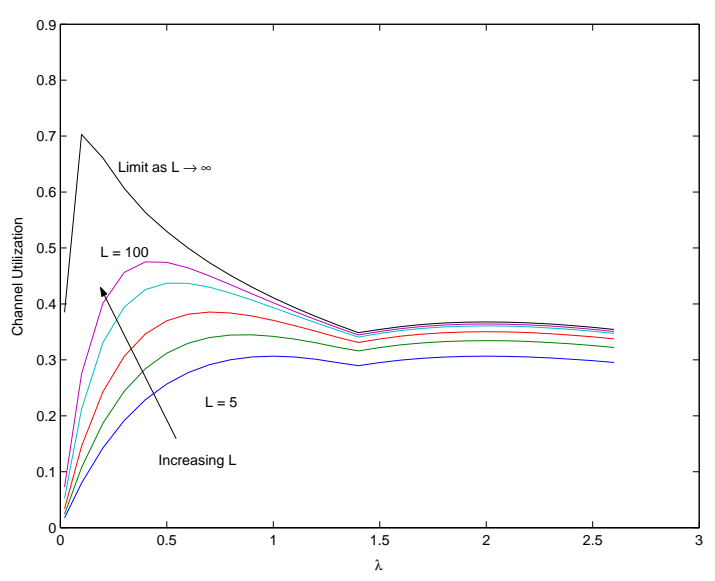

Fig. 4: Effect of increasing $L$ on channel utilization. Bayesian detector, $\mathrm{SNR}=0 \mathrm{~dB}$.

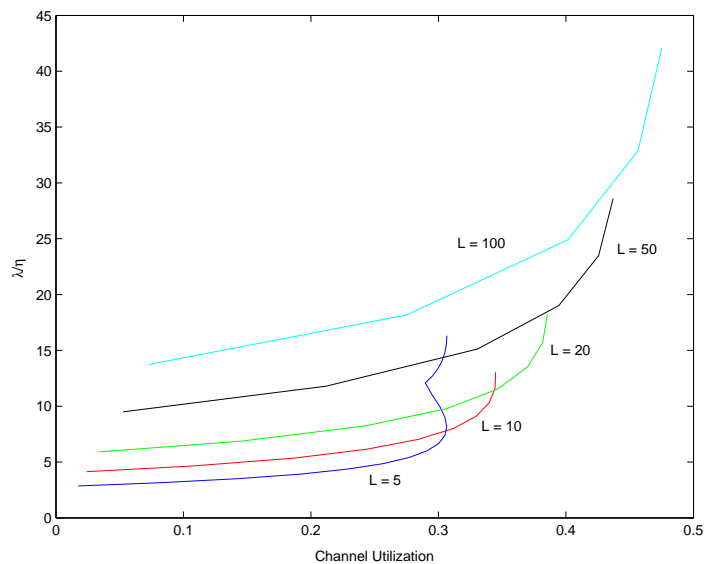

Fig. 5: Bayesian detector: Trade-off of channel utilization versus number of retransmissions required, $\mathrm{SNR}=0 \mathrm{~dB}$.

trade-off that exists between channel utilization and throughput for variations in $L$.

Plotted in Figs. 2 and 3 is the throughput for various detectors for various SNRs with $L$ equal to 10 . We see that the Bayesian detector optimized for cost gives the optimal performance throughout. This is to be expected, as the Bayesian detector utilizes every information it has and optimizes for the missing cost-ratios. For low SNR, the single threshold and UMP detectors are close to the optimal achievable. The performance of the multihypotheses MAP is not encouraging for low SNR, but for high SNR, it gives throughput close to that achieved using the Bayesian detector.

A higher throughput for the same arrival rate not only means higher channel utilization but also less number of retransmission attempts. The number of retransmissions required can serve as a measure of the delay incurred [4] and it is easy to see that the expected number of retransmissions is given by:

$$
R=\lambda / \eta
$$

We thus see that the Bayesian detector optimized for cost, not only maximizes the channel utilization given $L$ but also requires less number of retries.

From Fig. 4 it is evident that increasing $L$ leads to increased 


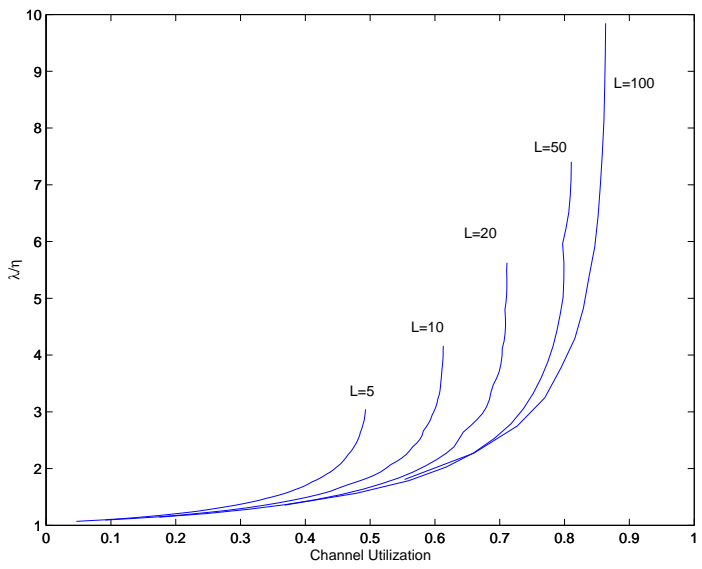

Fig. 6: Bayesian detector: Trade-off of channel utilization versus number of retransmissions required, $\mathrm{SNR}=20 \mathrm{~dB}$.

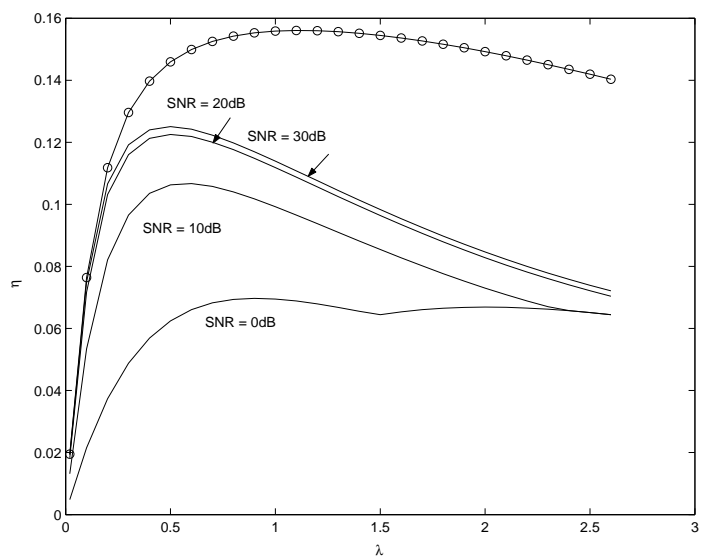

Fig. 7: Bayesian detector, increasing SNR, L = 10 (Ideal-o).

channel utilization. However, the channel utilization does not increase beyond a limit as computed in (10), and the limit is reached only gradually, as can be seen from the figure.

A comment here about the 'kinks' in the plots, conspicuous in Fig. 4 and also observed in the previous figures, would be in order. Though we do not have a satisfactory explaination for their presence, it is observed that as the arrival rate increases beyond that corresponding to the kink, the optimal policy is to always NACK when only one channel is free and always ACK when both channels are free. With such a policy, it can easily be checked that the local maximum occurs at $\lambda=2$ (in which case, with two channels free the arrival rate per channel is $\lambda_{2}=1$ ).

Figs. 5 and 6 depict the trade-off that exists between channel utilization and the number of retransmissions required. The trade-off is especially severe when the SNR is low, higher channel utilization coming at the price of increased number of retransmissions required and therefore, incurring more delay. The trade-off is almost non-existent for higher SNR, though the channel utilization peaks for a lower offered rate, $\lambda$, as is seen in Fig.4.

As we commented in Sec.IVF, the throughput saturates with increasing SNR, as seen in Figs.7 and 8. With increased number of measurements, throughput close to the ideal can be reached.

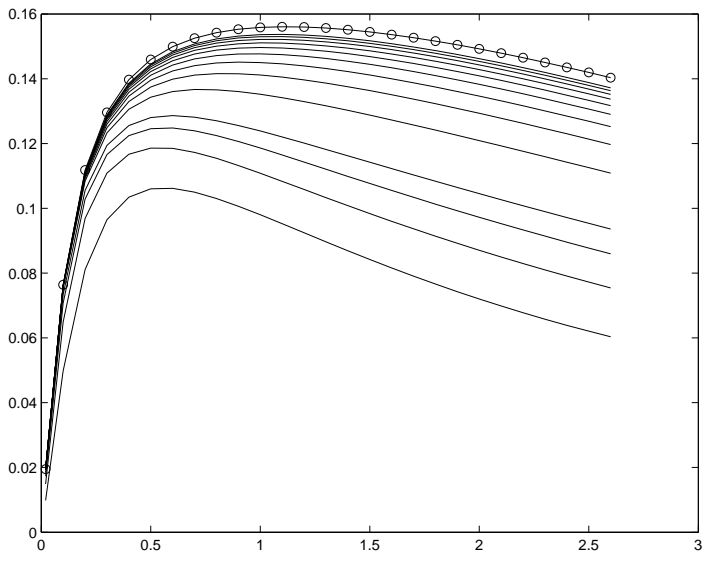

Fig. 8: Multihypotheses, increasing measurements (1,2,3,4:4:40), $\mathrm{SNR}=10, \mathrm{~L}=10$ (Ideal-o)

\section{CONCLUSION}

For a system employing reservation for multi-access over multiple channels, we have given a framework wherein the performance at MAC level can be analyzed under channel fading conditions in which detectors cannot be assumed to perform perfectly. We have presented detectors which will lead to optimal performance under the said conditions. The optimal detectors are Bayesian detectors optimized for cost and have to be searched for numerically. These detectors utilize all the available information, including traffic statistics and system state, in determining the power thresholds for channel acknowledgement. The performance of these detectors can also be used as a benchmark in evaluating detectors which may not be able to use the entire information in making decisions.

\section{References}

[1] H.V.Poor, An Introduction to Signal Detection and Estimation, Springer-Verlag, New York, 1994

[2] D.P. Bertsekas and R. Gallager, Data Networks, Prentice Hall, 2nd Edition, 1992

[3] I.N.Vukovic and T. Brown, "Performance Analysis of the Random Access Channel (RACH) in WCDMA", in Proc. of Vehicular Tech. Conf.,pp.532-536, Spring 2001

[4] L. Kleinrock and F.A. Tobagi, "Packet Switching in Radio Channels: Part I-Carrier Sensing Multiple-Access Modes and Their Throughput-Delay Characteristics", IEEE Trans. Comm., COM-23, pp.1400-1416, Dec. 1975.

[5] E.L. Lehmann, Testing Statistical Hypotheses, John Wiley \& Sons, Inc., New York, NY, 1959.

[6] TS 25.321, $3^{\text {rd }}$ Generation Partnership Project, MAC Protocol Specification, available on http://www.etsi.org

[7] L.G. Alonso, R. Agusti, O. Sallent, "A new Medium Access Protocol based on Distributed Queues for a CDMA Environment", in Proc. of Vehicular Tech. Conf., pp.11751179,1999

[8] A. Maharshi, L. Tong, A. Swami, "Detection for Medium Access Control in Random Access CDMA", in Proc. 3xth Conference of Information Science and Systems, Princeton, NJ, March 2002. 